\title{
Prognostic and clinicopathological significance of circulating tumor cells detected by RT-PCR in non-metastatic colorectal cancer: a meta-analysis and systematic review
}

\author{
Chaogang Yang ${ }^{1+}$, Kun Zou $^{2+}$, Liang Zheng ${ }^{1}$ and Bin Xiong ${ }^{1 *}$
}

\begin{abstract}
Background: Circulating tumor cells (CTCs) have been accepted as a prognostic marker in patients with metastatic colorectal cancer (mCRC, UICC stage IV). However, the prognostic value of CTCs in patients with non-metastatic colorectal cancer (non-mCRC, UICC stage I-III) still remains in dispute. A meta-analysis was performed to investigate the prognostic significance of CTCs detected by the RT-PCR method in patients diagnosed with non-mCRC patients.

Methods: A comprehensive literature search for relevant articles was performed in the EmBase, PubMed, Ovid, Web of Science, Cochrane library and Google Scholar databases. The studies were selected according to predetermined inclusion/exclusion criteria. Using the random-effects model of Stata software, version12.0 (2011) (Stata Corp, College Station, TX, USA), to conduct the meta-analysis, and the hazard ratio (HR), risk ratio (RR) and their $95 \%$ confidence intervals $(95 \% \mathrm{Cls})$ were regarded as the effect measures. Subgroup analyses and meta-regression were also conducted to clarify the heterogeneity.

Results: Twelve eligible studies, containing 2363 patients with non-mCRC, were suitable for final analyses. The results showed that the overall survival (OS) (HR=3.07, 95\% Cl: $[2.05-4.624], P<0.001 ; I^{2}=55.7 \%$, $P=0.008)$ and disease-free survival (DFS) (HR=2.58,95\% Cl: $\left.[2.00-3.32], P<0.001 ; I^{2}=34.0 \%, P=0.085\right)$ were poorer in patients with CTC-positive, regardless of the sampling time, adjuvant therapy and TNM stage. CTC-positive was also significantly associated with regional lymph nodes (RLNs) metastasis (RR $=1.62$, 95\% Cl: $\left.[1.17-2.23], P=0.003 ; I^{2}=74.6 \%, P<0.001\right)$, depth of infiltration $(R R=1.41,95 \% C l:[1.03-1.92], P=0.03$; $\left.I^{2}=38.3 \%, P=0.136\right)$, vascular invasion (RR $\left.=1.66,95 \% \mathrm{Cl}:[1.17-2.36], P=0.004 ; I^{2}=46.0 \%, P=0.135\right)$, tumor grade $\left(\mathrm{RR}=1.19,95 \% \mathrm{Cl}:[1.02-1.40], P=0.029 ; I^{2}=0 \%, P=0.821\right)$ and tumor-node-metastasis (TNM) stage(I, II versus III) $\left(\mathrm{RR}=0.76,95 \% \mathrm{Cl} 0.71-0.81, P<0.001 ; I^{2}=0 \%, P=0.717\right)$. However, there was no significant relationship between CTC-positive and tumor size (RR $\left.=1.08,95 \% \mathrm{Cl}:[0.94-1.24], P=0.30 ; I^{2}=0 \%, P=0.528\right)$.

(Continued on next page)
\end{abstract}

\footnotetext{
* Correspondence: binxiong1961@whu.edu.cn

${ }^{\dagger}$ Equal contributors

'Department of Gastrointestinal Surgery \& Department of Gastric and Colorectal Surgical Oncology, Zhongnan Hospital of Wuhan University; Hubei Key Laboratory of Tumor Biological Behaviors \& Hubei Cancer Clinical Study Center, No.169 Donghu Road, Wuchang District, Wuhan 430071, China Full list of author information is available at the end of the article
} 
(Continued from previous page)

Conclusions: Detection of CTCs by RT-PCR method has prognostic value for non-mCRC patients, and CTC-positive was associated with poor prognosis and poor clinicopathological prognostic factors. However, the prognostic value of CTCS supports the use of CTCs as an indicator of metastatic disease prior to the current classification of mCRC meaning it is detectable by CT/MRI.

Keywords: Circulating tumor cells, Non-metastatic colorectal cancer, RT-PCR, Prognosis, Meta-analysis

\section{Background}

Colorectal cancer (CRC) is the third most commonly diagnosed cancer and the fourth leading cause of cancerrelated death [1]. In China, CRC is ranked fourth in morbidity and mortality among the gastrointestinal cancers [2]. Due to the difficulties of early diagnosis, a large proportion of patients with CRC are undiagnosed until an advanced stage. Due to the continuous improvement of the treatment methods, decreasing CRC mortality rates have been observed in a large number of countries worldwide [3], especially for the patients with nonmetastatic colorectal cancer (non-mCRC, UICC I-III). Unfortunately, the 5-year overall survival (OS) of nonmCRC patients is still low and approximately $25-50 \%$ of patients with stage II-III CRC will experience recurrence or distant metastasis after comprehensive treatment [4], which is the main reason for studying the prognosis in those patients. The mechanisms of recurrence and metastasis of CRC are very complicated and remains unclear. Recurrence and metastasis may involve series of cell biological behaviors, including circulating tumor cells (CTCs), which have been gradually recognized to play an important role in the process of distant metastasis, according to the "seed and soil theory" [5].

CTCs, which were defined as the "break away" cancer cells in the peripheral blood (PB) of cancer patients, were firstly proposed by Ashworth in1869 [6] and further demonstrated by Engell in 1955 [7]. These cells, which shed intermittently from the solid tumors, circulate in the bloodstream, and arrive at different positions, are the main cause of distant metastases [8]. However, the lower concentration of $\mathrm{PB}$ in the solid tumors, which are confined to local growth $[9,10]$, makes it difficult to detect in early CRC. During the past few decades, with a variety of highly sensitive and specific diagnostic approaches including reverse transcriptase-polymerase chain reaction (RT-PCR), immunocytochemistry, flow cytometry, and the CellSearch system, the efficiency of detecting CTCs is increasing gradually. Encouraging results from numerous studies have demonstrated that the presence of CTCs was significantly associated with poor prognosis of CRC patients. However, most large-scale data were collected from patients with mCRC [11, $12]$, there were only limited data on the significance of CTC in patients with non-mCRC. In those studies, the diagnostic method used to detect CTCs was predominantly the CellSearch system [13-15], which is the first and only method approved by the US Food and Drug Administration (FDA) for evaluating the prognosis of CRC patients [16]. However, while there are advantages of high specificity and reproducibility for CTC detection, as a semi-automated system, CellSearch has the disadvantages of moderate sensitivity and subjective verification. Compared to CellSearch, RT-PCR has higher sensitivity and is more objective for detection of CTCs $[17,18]$. Therefore, it has also been widely used for the detection of CTCs for non-mCRC patients, and the clinical utility has been demonstrated in several studies. Shimada et al. reported that CTCs detection with the RT-PCR method was correlated with tumor metastasis and prognosis [19]. However, Kust et al. showed that CTCs detected with RT-PCR had unfavorable prognostic significance for non-mCRC patients [20]. Therefore, the prognostic role of CTC detection with RT-PCR in non-mCRC is still controversial.

We performed a pooled analysis of published studies to quantitatively and comprehensively summarize the prognostic relevance of CTCs detected by RT-PCR in patients with non-mCRC.

\section{Methods}

\section{Search strategy}

A literature search for relevant studies was performed systematically from the EmBase, PubMed, Ovid, Web of Science, Cochrane library and Google Scholar database with key words "colorectal cancer", "circulating tumor cells" or "CTCs" and "polymerase chain reaction or PCR" by two researchers (CG Yang and K Zou) independently (up to July, 2016). No time restriction was imposed. In order to prevent missing relevant studies, "related articles" function of PubMed and Google Scholar were used to identify other potentially relevant publications.

\section{Inclusion and exclusion criteria}

The inclusion criteria for our meta-analysis were: (1) investigated the clinicopathological or prognostic significance of CTC detection in non-mCRC patients; (2) used any form of RT-PCR for detecting CTCs; (3) hazard ratio $(\mathrm{HR})$ or a risk ratio( $\mathrm{RR})$ with a $95 \%$ confidence 
interval $(95 \% \mathrm{CI})$ of $\mathrm{OS}$ or/and disease-free survival (DFS) reported in the study or had sufficient data to calculate; (4) collected the samples from PB. Exclusion criteria were: (1) studies including mCRC patients; (2) the number of patients was less than 20. (3) exclusion of letters, reviews, and articles published with non-English language. (4) the study was redundant, based on the same database or patient population as an included study. To avoid the inclusion of redundant studies, all the included studies were checked carefully, including their authors, organizations, accrual period, and population of patients.

\section{Data extraction and quality assessment}

Two reviewers (CG Yang and K Zou) evaluated the quality of the included studies and extracted data independently. The following information was collected: first author, year of publication, country, characteristics of the study population (number, sex and age), TNM stage (UICC), detection markers, adjuvant therapy, sampling time (pre/intra/post-operation), rate of CTC positivity rate, follow-up period, the $\mathrm{HR}$ and their associated standard errors on prognostic outcomes (OS or/and DFS). If the HRs and its 95\% CI were not directly provided in the original articles, we used the method designed by Jayne F. Tierney [21] to calculate them from the available data. In addition, when HRs were presented by both univariate and multivariate analyses, the latter ones were preferable because multivariate analyses also considered possible confounding of exposure effects [22]. The quality assessment was based on the Newcastle-Ottawa Scale (NOS) criteria, which is recommended by the Cochrane Library for the cohort study, score $5-9$ is considered as high quality and $1-4$ is low quality [23]. The results of quality assessment and data extraction were confirmed by two reviewers. Any disagreements about data extraction and quality assessment were resolved by comprehensive discussion and were checked by the third investigator.

\section{Statistical analysis}

Statistical analyses were implemented with Stata software, version 12.0 (2011) (Stata Corp, College Station, TX, USA). The RR and HR were regarded as effect measures for summarizing the clinicopathological and prognostic significance of CTCs detected by RT-PCR in non-mCRC. By convention, a HR $>1$ indicates a poorer prognosis in the CTC-positive group in contrast with negative group and a RR $>1$ implies CTCpositive be associated with a parameter. All statistical values were reported with $95 \%$ confidence intervals (95\% CIs) and $P$ value $<0.05$ was considered statistically significant. To retain maximum information, we added additional information into included study from original authors or excluded studies if the included and excluded studies were based on the same patients' population and some information of interest was reported in the excluded studies but not in the included studies. All relevant studies were included in the overall analysis. Subgroup analyses were performed based on the sampling time (pre/intra/post$\mathrm{OP})$, TNM stage (II/III), adjuvant therapy (without/ post-OP chemotherapy) and detection markers (single/multiple). All data analyses used a random effects model, because it provided more conservative estimates and more tailored to multicenter studies in which heterogeneity was usually present [24]. The Cochrane's $\mathrm{Q}$ statistic and $\mathrm{I}^{2}$ statistic were applied to evaluate the heterogeneity among studies. $P$ value $<0.01$ for the $Q$ statistic and/or $\mathrm{I}^{2}>50 \%$ were considered significant heterogeneity [25]. The $\mathrm{I}^{2}$ value indicated the degree of heterogeneity. Potential heterogeneity between-study was illustrated by forest plots. If necessary, meta-regression was performed to explore the potential source of heterogeneity. Lastly, we evaluated potential publication bias by a funnel plot, which was further validated by the Egger [26] and Begg's test [27].

\section{Results}

\section{Baseline characteristics of the eligible studies}

Initially, 206 relevant studies were identified in the systematic literature search process. By checking the titles and abstracts, 164 studies were excluded and 42 potential studies were retrieved. An additional 30 studies were then excluded after they were fully reviewed because they lacked sufficient data (2 studies), were redundant (2 studies), or included stage IV patients (26 studies). Finally, 12 studies were yielded as meeting our inclusion criteria and were eligible for our meta-analysis (Fig. 1).

Twelve eligible studies, including 23 sets of data, contained 2363 patients with non-mCRC [19, 20, 28-37]. The studies were conducted in seven countries (Australia, China, Croatia, Germany, Japan, Spain and the UK) and were published between 2002 and 2016. All studies detected tumor cells from $\mathrm{PB}$ with the molecular detection method (PCR, RT-PCR, or RT followed by quantitative PCR). Table 1 summarizes the main baseline characteristics and study design variables. The quality of the eligible cohort studies was assessed with NOS and is summarized in Table 2.

\section{Effects of CTCs on OS and DFS for non-mCRC patients}

Data on OS were available in 13 sets of data included in eight studies $[19,20,28-30,33,35,36]$. The pooled analysis showed CTC-positive was significantly associated with a poor OS $(\mathrm{HR}=3.07,95 \% \mathrm{CI}$ : [2.05-4.624], $P<0.001$ ), with significant between-study heterogeneity $\left(\mathrm{I}^{2}=55.7 \%, P=0.008\right.$; Fig. $\left.2 \mathrm{a}\right)$ in non-mCRC patients. 


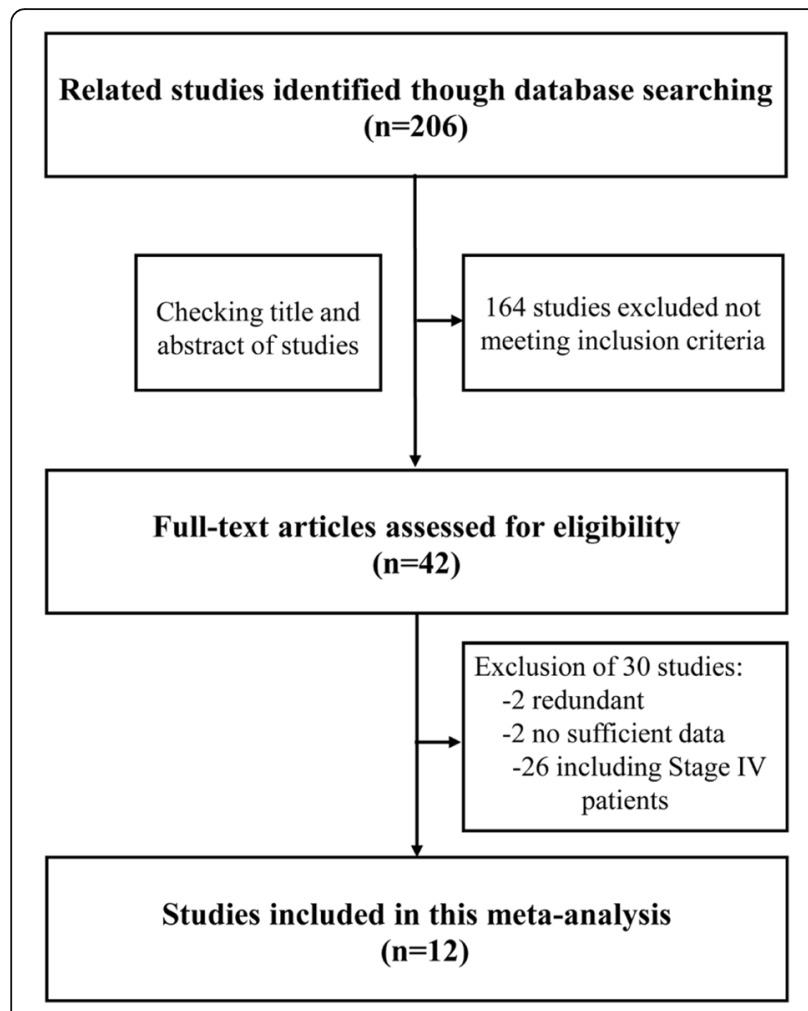

Fig. 1 Flow chart showing the selection process for the included studies

Seventeen sets of data included in all enrolled studies contained the data on DFS [19, 20, 28-37]; the pooled analysis indicated CTC-positive was also associated with a significantly decreased DFS $(\mathrm{HR}=2.58$, 95\% CI: [2.00-3.32], $P<0.001)$ with no between-study heterogeneity $\left(\mathrm{I}^{2}=34.0 \%, P=0.085\right.$; Fig.

2b). To further investigate the effect of CTCs detection on the prognosis of non-mCRC patients under different conditions, subgroup analyses were performed based on different sampling time (pre-OP and intra/post-OP), TNM stage (II/III) and adjuvant therapy status (without/post-OP chemotherapy). The results demonstrated CTC-positive was significantly associated with poor OS (HR $=3.65$, 95\% CI: [2.495.36], $P<0.001 ; \mathrm{HR}=2.44,95 \%$ CI: [1.19-4.99], $P=$ 0.015 ; Fig. 3a) and DFS (HR $=3.08$, 95\% CI: [2.214.31], $P<0.001$; HR $=2.23$, 95\% CI: [1.50-3.29], $P<0.001$; Fig. $3 \mathrm{~b}$ ) in non-mCRC patients, regardless of pre-OP or intra/post-OP sample collection. Furthermore, due to the limited number of studies on about neoadjuvant radiotherapy or/and chemotherapy and post-OP adjuvant radiotherapy in the included studies, we conducted a subgroup analysis to evaluate to prognostic value of CTCs in patients who did and did not receive post-operative chemotherapy. The results showed no difference between these two groups (OS, $\mathrm{HR}=2.96,95 \% \mathrm{CI}$ :
[1.96-4.47], $P<0.001 ; \mathrm{HR}=3.59,95 \%$ CI: [2.26-5.71], $P=0.015$; Fig. 3c. DFS, $\mathrm{HR}=2.83$, 95\% CI: [1.924.19], $P<0.001 ; \mathrm{HR}=3.19,95 \%$ CI: [2.26-4.50], $P<$ 0.001 ; Fig. $3 \mathrm{~d}$ ). For TNM stage, subgroup analyses were only performed to explore the prognostic value of CTCs for stage II and III CRC patients; the results demonstrated that CTC-positive was significantly associated with poor OS $(\mathrm{HR}=3.72,95 \% \mathrm{CI}$ : [2.36-5.85], $P<0.001$; HR $=2.94,95 \%$ CI: [2.09-4.14], $P<0.001$; Fig. 3e) and DFS $(\mathrm{HR}=2.77,95 \% \mathrm{CI}:[1.90-4.02], P<0.001 ; \mathrm{HR}=3.00$, 95\% CI: [2.19-4.11], $P<0.001$; Fig. 3f) for both stage II and III CRC patients.

\section{Association between CTCs and clinicopathological parameters}

Seven studies [19, 28, 29, 31, 35-37] including eight sets of data were evaluated to determine the relationship between CTC-positive and regional lymph nodes metastasis. The results showed regional lymph nodes metastasis was associated with CTC-positive $(R R=1.62$, 95\% CI: [1.17-2.23], $P=0.003)$ with significant betweenstudy heterogeneity $\left(I^{2}=74.6 \%, P<0.001\right.$; Fig. $\left.4 a\right)$. The depth of tumor infiltration was associated with CTC-positive (RR $=1.41,95 \%$ CI: $[1.03-1.92], P=0.03 ; \mathrm{I}^{2}=38.3 \%, P$ $=0.136$; Fig. 4b). Studies assessed by pooled analysis showed significant association between CTC-positive and vascular invasion $(\mathrm{RR}=1.66,95 \% \mathrm{CI}$ : $[1.17-2.36], P=$ $0.004 ; \mathrm{I}^{2}=46.0 \%, P=0.135$; Fig. $\left.4 \mathrm{c}\right)$. Eight sets of data from seven studies $[19,28,29,31,35-37]$ demonstrated that tumor grade was associated with CTC-positive $(\mathrm{RR}=$ 1.19, 95\% CI: [1.02-1.40], $P=0.029 ; \mathrm{I}^{2}=0 \%, P=0.821$; Fig. 4d). Eight studies [19, 20, 28, 29, 31, 35-37] reported the relationship between CTC-positive and TNM stage (I, II versus III). As shown in Fig. 3e, CTC-positive in stage III is greater than in stage I and II $(R R=0.76$, 95\% CI: [0.71-0.81], $P<0.001 ; \mathrm{I}^{2}=0 \%, P=0.717$; Fig. $4 \mathrm{e})$. Furthermore, the pooled analysis found no significant relationship between CTC-positive and tumor size $\left(\mathrm{RR}=1.08,95 \% \mathrm{CI}\right.$ : [0.94-1.24], $P=0.30 ; \mathrm{I}^{2}=0 \%, P=$ 0.528; Fig. 4f).

\section{Exploring the sources of heterogeneity}

To examine the intra-study inconsistencies on OS, we stratified the eligible studies according to variables as shown in Table 3 . The pooled analyses results showed the heterogeneity did not drop to an insignificant level, regardless of the variables. Therefore, meta-regression was further implied to explore the source of heterogeneity on OS. As shown in Table 4, for the studies on OS, only positive rate of CTC detection was significantly correlated with intra-study variability $(P=0.021)$, and it explained $93.8 \%$ of the between-study variance in the multivariate analysis. 


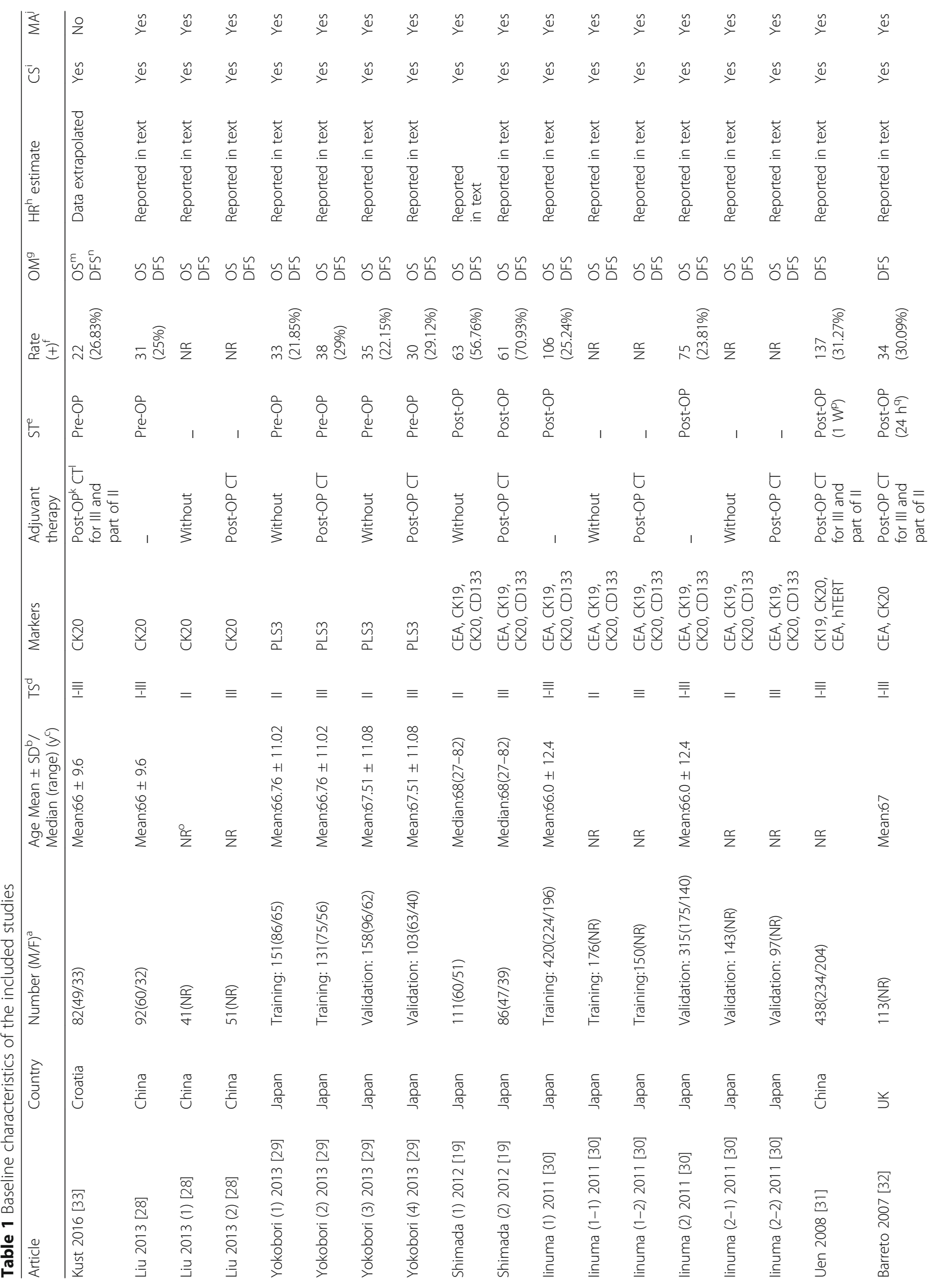




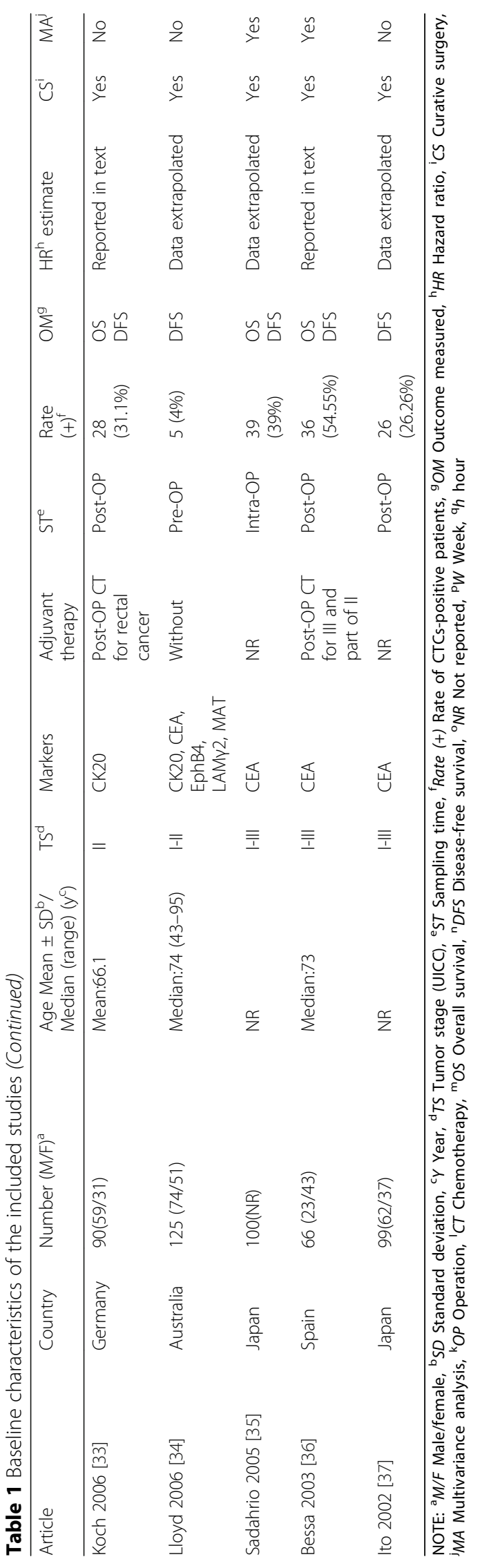


Table 2 The assessment of the risk of bias in each Cohort study using the Newcastle-Ottawa Scale

\begin{tabular}{|c|c|c|c|c|c|c|c|c|c|c|}
\hline \multirow[t]{2}{*}{ Study } & \multicolumn{4}{|c|}{ Selection (0-4) } & \multicolumn{2}{|c|}{ Comparability (0-2) } & \multicolumn{2}{|c|}{ Outcome $(0-3)$} & \multirow{2}{*}{$\frac{\text { Total }}{\text { AFU }}$} & \\
\hline & REC & SNEC & $\mathrm{AE}$ & $\mathrm{DO}$ & SC & $\mathrm{AF}$ & $\mathrm{AO}$ & FU & & \\
\hline Kust 2016 [20] & 0 & 1 & 1 & 1 & 0 & 0 & 0 & 1 & 0 & . \\
\hline Liu 2013 [28] & 0 & 1 & 1 & 1 & 0 & 0 & 1 & 1 & 0 & \\
\hline Yokobori 2013 [29] & 1 & 1 & 1 & 1 & 0 & 0 & 1 & 1 & 0 & . \\
\hline Shimada 2012 [19] & 1 & 1 & 1 & 1 & 0 & 0 & 1 & 1 & 1 & . \\
\hline limuna 2011 [30] & 0 & 1 & 1 & 1 & 0 & 0 & 1 & 1 & 0 & . \\
\hline Uen 2008 [31] & 0 & 1 & 1 & 1 & 0 & 0 & 1 & 1 & 0 & . \\
\hline Barreto 2007 [32] & 0 & 0 & 1 & 1 & 0 & 0 & 1 & 1 & 0 & \\
\hline Koch 2006 [33] & 0 & 1 & 1 & 1 & 0 & 0 & 1 & 0 & 0 & \\
\hline Lloyd 2006 [34] & 0 & 0 & 1 & 1 & 0 & 0 & 1 & 0 & 0 & \\
\hline Sadahrio 2005 [35] & 1 & 1 & 1 & 1 & 0 & 0 & 1 & 1 & 1 & \\
\hline Bessa 2003 [36] & 0 & 1 & 1 & 1 & 0 & 0 & 1 & 1 & 0 & \\
\hline Ito 2002 [37] & 0 & 1 & 1 & 1 & 0 & 0 & 1 & 1 & 0 & \\
\hline
\end{tabular}

NOTE: REC representativeness of the exposed cohort, SNEC selection of the non-exposed cohort, $A E$ ascertainment of exposure; DO: demonstration that outcome of interest was not present at start of study, SC study controls for age, sex, $A F$ study controls for any additional factors (chemoradiotherapy, curative resection), $A O$ assessment of outcome, FU follow-up long enough ( $36 \mathrm{M})$ for outcomes to occur, AFU adequacy of follow-up of cohorts ( $\geq 90 \%)$.Total: the points of each study

\section{Publication bias}

Potential publication bias was assessed by Begg's and Egger's tests. $P<0.05$ indicated the existence of publication bias. There was no evidence of publication bias for the pooled analysis of OS $\left(P_{\text {Begg }}=0.246, P_{\text {Egger }}=0.964\right)$ and DFS $\left(P_{\text {Begg }}=0.434, P_{\text {Egger }}=0.301\right)$. The funnel plots of publication bias on OS and DFS are shown in Fig. 5a and b, respectively.

\section{Discussion}

Currently, the treatment strategies for non-mCRC include radical surgery as well as neoadjuvant and adjuvant radio-chemotherapy. In clinical practice, the oncologist selects the most appropriate regiment depending on the TNM stage, which is based on the extent of tumor invasion $(\mathrm{T})$, the presence of metastases or micro-metastases in regional lymph nodes $(\mathrm{N})$ and distant metastases (M) [38]. The clinical TNM stage, which is based on the imaging examination, can help oncologists assess whether neoadjuvant radio-chemotherapy should be incorporated before surgery, whereas the pathological TNM stage, which is based on the histopathologic examination of post-operative samples, provides information on whether adjuvant radio-chemotherapy should be included after curative resection. Despite advances in therapeutic approaches, it is estimated that approximately $30 \%$ of patients will develop metastases and eventually succumb to their disease after comprehensive treatment [39]. In general, the prognosis outcome of non-mCRC patients is directed by the TNM-stage, which provides the

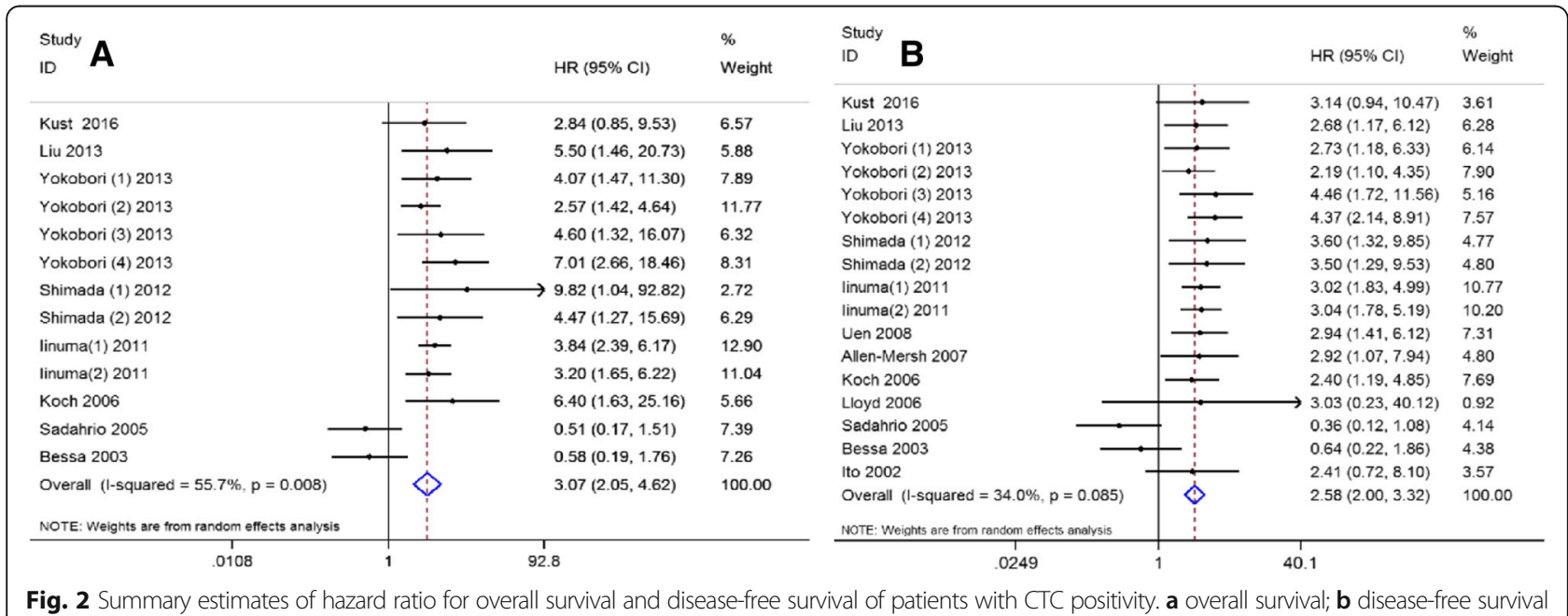




\begin{tabular}{|c|c|c|c|c|c|c|c|}
\hline${ }_{\text {ID }}^{\text {Study }} \mathbf{A}$ & & $\mathrm{HR}(95 \% \mathrm{Cl})$ & ${ }_{\text {Weight }}$ & ${ }_{\text {ID }}^{\text {Study }} \mathbf{B}$ & & $\mathrm{HR}(95 \% \mathrm{Cl})$ & $\stackrel{\%}{\text { Weight }}$ \\
\hline Pre-op & & & & $\begin{array}{l}\text { Pre-OP } \\
\text { Kust } 2016\end{array}$ & & $3.14(0.94,10.47)$ & 3.61 \\
\hline Kust 2016 & $\div$ & $2.84(0.85,9.53)$ & 6.57 & Liu 2013 & - & $2.68(1.17,6.12)$ & 6.28 \\
\hline Liu 2013 & $\longrightarrow$ & $5.50(1.46 .20 .73)$ & 5.88 & Yokobori (1) 2013 & $\longrightarrow$ & $2.73(1.18,6.33)$ & 6.14 \\
\hline Yokobori (1) 2013 & $\hookrightarrow$ & $4.07(1.47 .11 .30)$ & 7.89 & Yokobori (2) 2013 & $\because$ & 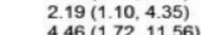 & 7.90 \\
\hline Yokobori (2) 2013 & + & $2.57(1.42,4.64)$ & 11.77 & $\begin{array}{l}\text { Yokobori (3) } 2013 \\
\text { Yokobori (4) } 2013\end{array}$ & $+\square$ & $\begin{array}{l}4.46(1.72,11.56) \\
4.37(2.14,8.91)\end{array}$ & $\begin{array}{l}5.16 \\
7.57\end{array}$ \\
\hline $\begin{array}{l}\text { Yokobori (3) } 2013 \\
\text { Yokobori (4) } 2013\end{array}$ & $\square$ & $\begin{array}{l}4.60(1.32,16.07) \\
7.01(2.66,18.46)\end{array}$ & 6.32 & $\begin{array}{l}\text { Yokobori (4) } 2013 \\
\text { Loyd 2006 }\end{array}$ & & $3.03(0.23,40.12)$ & 0.92 \\
\hline $\begin{array}{l}\text { Yokobori (4) } 2013 \\
\text { Subtotal }(1-\text { squared }=0.0 \%, p=0.578 \text { ) }\end{array}$ & $\infty$ & $\begin{array}{l}7.01(2.66,18.46) \\
3.65(2.49 .5 .36)\end{array}$ & $\begin{array}{l}8.31 \\
46.74\end{array}$ & Subtotal (l-squared $=0.0 \%, p=0.852$ ) & 0 & $3.08(2.21,4.31)$ & 37.58 \\
\hline Intra/post-op & & & & $\begin{array}{l}\text { Intra/post-OP } \\
\text { Shimada (1) } 2012\end{array}$ & 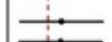 & $3.60(1.32,9.85)$ & 4.77 \\
\hline $\begin{array}{l}\text { Shimada (1) } 2012 \\
\text { Shimada (2) } 2012\end{array}$ & 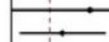 & $\begin{array}{l}9.82(1.04,92.82) \\
4.47(1.27,15.69)\end{array}$ & $\begin{array}{l}2.72 \\
6.29\end{array}$ & $\begin{array}{l}\text { Shimada (2) } 2012 \\
\text { linuma(1) } 2011\end{array}$ & $\div$ & $\begin{array}{l}3.50(1.29,9.53) \\
3.02(1.83,4.99)\end{array}$ & $\begin{array}{l}4.80 \\
10.77\end{array}$ \\
\hline $\begin{array}{l}\text { Shimada (2) } 2012 \\
\text { linuma(1) } 2011\end{array}$ & $\div$ & $\begin{array}{l}4.47(1.27,15.69) \\
3.84(2.39,6.17)\end{array}$ & $\begin{array}{l}6.29 \\
12.90\end{array}$ & linuma(2) 2011 & $\stackrel{\div}{\square}$ & $3.04(1.78,5.19)$ & 10.20 \\
\hline linuma(2) 2011 & $\rightarrow$ & $3.20(1.65,6.22)$ & 11.04 & $\begin{array}{l}\text { Uen } 2008 \\
\text { Allen-Mersh } 2007\end{array}$ & 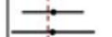 & $2.94(1.41,6.12)$ & $\begin{array}{l}7.31 \\
480\end{array}$ \\
\hline Koch 2006 & $\longrightarrow$ & $6.40(1.63,25.16)$ & 5.66 & $\begin{array}{l}\text { Alien-Merss } 20006 \\
\text { Koch } 2006\end{array}$ & $\square$ & $2.40(1.19,4.85)$ & $\begin{array}{l}4.80 \\
7.69\end{array}$ \\
\hline Sadahrio 2005 & $-:$ & $0.51(0.17,1.51)$ & 7.39 & Sadahrio 2005 & & $0.36(0.12,1.08)$ & 4.14 \\
\hline Bessa 2003 & & $0.58(0.19,1.76)$ & 7.26 & Bessa 2003 & + & $0.64(0.22,1.86)$ & 4.38 \\
\hline Subtotal (1-squared $=72.9 \%, p=0.001)$ & $\infty$ & $2.44(1.19 .4 .99)$ & 53.26 & $\begin{array}{l}\text { Ito } 2002 \\
\text { Subtotal (1-squared }=55.9 \%, p=0.016 \text { ) }\end{array}$ & $\Delta$ & $\begin{array}{l}2.41(0.72,8.10) \\
2.23(1.50,3.29)\end{array}$ & $\begin{array}{l}3.57 \\
62.42\end{array}$ \\
\hline $\begin{array}{l}\text { Overall (l-squared }=55.7 \%, p=0.008 \text { ) } \\
\text { NOTE: Weights are from random effects anaysis }\end{array}$ & $\phi$ & $3.07(2.05,4.62)$ & 100.00 & $\begin{array}{l}\text { Overall (1-squared }=34.0 \%, p=0.085 \text { ) } \\
\text { NOTE Weights aro trom random eftects analysis }\end{array}$ & 0 & $2.58(2.00,3.32)$ & 100.00 \\
\hline .0108 & 1 & 92.8 & & .0249 & 1 & 40.1 & \\
\hline${ }_{{ }^{10}}^{\text {Study }} \mathbf{C}$ & & HR $(95 \% \mathrm{Cl})$ & $\begin{array}{l}\% \\
\text { Weight }\end{array}$ & ${ }_{\text {ID }}^{\text {Study }} \mathbf{D}$ & & $\operatorname{HR}(95 \% \mathrm{CI})$ & $\begin{array}{l}\% \\
\text { Weight }\end{array}$ \\
\hline $\begin{array}{l}\text { Without } \\
\text { Lif (1) } 2013\end{array}$ & & & & Without & & & \\
\hline $\begin{array}{l}\text { Liu (1) } 2013 \\
\text { Yokobori(1)2013 }\end{array}$ & 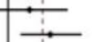 & $2.07(0.58,7.32)$ & 4.77 & $\begin{array}{l}\text { Liu (1) } 2013 \\
\text { Yokobori (1) } 2013\end{array}$ & & $\begin{array}{l}2.47(1.02,5.96) \\
2.73(1.16 .6 .24)\end{array}$ & 7.08 \\
\hline $\begin{array}{l}\text { Yokobori(1)2013 } \\
\text { Yokobori(3)2013 }\end{array}$ & 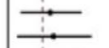 & $4.07(1.50,11.57)$ & 7.34 & & & $\begin{array}{l}2.73(1.16,6.24) \\
4.46(1.73,11.62)\end{array}$ & 7.80 \\
\hline $\begin{array}{l}\text { Yokobori(3)22013 } \\
\text { Shimada (1) } 2012\end{array}$ & $\square$ & $4.60(1.34,16.36)$ & 4.90 & $\begin{array}{l}\text { Yokobori (3) } 2013 \\
\text { Shimada (1) } 2012\end{array}$ & ه. & $\begin{array}{l}4.46(1.73,11.62) \\
360(1.45,1086)\end{array}$ & 6.08 \\
\hline $\begin{array}{l}\text { Shimada (1) } 2012 \\
\text { linuma(1-1) } 2011\end{array}$ & & $9.82(1.99,177.57)$ & 1.52 & & 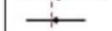 & $\begin{array}{l}3.60(1.45,10.86) \\
3.35(1.66 .64)\end{array}$ & $\begin{array}{l}5.46 \\
11.24\end{array}$ \\
\hline $\begin{array}{l}\text { linuma(1-1) } 2011 \\
\text { linumaa(2-1) 2011 }\end{array}$ & $\dot{i}$ & $3.21(1.44,7.46)$ & 11.33 & $\begin{array}{l}\text { linuma(1)-1) } 2011 \\
\text { linuma(2-1) } 2011\end{array}$ & ?: & $3.10(1.33,7.24)$ & $\begin{array}{l}7.24 \\
7.69\end{array}$ \\
\hline $\begin{array}{l}\text { linuma(2-1) } 2011 \\
\text { Subtotal }(1-\text { squared }=0.0 \%, p=0.879)\end{array}$ & $\dot{0}$ & $3.75(1.25,12.47)$ & 5.79 & Loyd 2006 & & $3.03(0.23,40.33)$ & 0.83 \\
\hline & & $3.59(2.26,5.71)$ & 35.65 & Subtotal (1-squared $=0.0 \%, p=0.985$ ) & & $3.19(2.26,4.50)$ & 46.18 \\
\hline $\begin{array}{l}\text { Post-OP CT } \\
\text { Liu (2) } 2013\end{array}$ & + & $0.52(0.06 .4 .68)$ & 1.61 & Post-OP CT & & & \\
\hline Yokobori(2)2013 & $\rightarrow$ & $2.57(1.42,4.63)$ & $\begin{array}{l}1.61 \\
21.94\end{array}$ & $\begin{array}{l}\text { Liu (2) } 2013 \\
\text { Yokobori (2) } 2013\end{array}$ & - & $0.51(0.11,2.42)$ & 2.31 \\
\hline Yokobori(4)22013 & 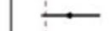 & $7.01(2.80,19.41)$ & 8.18 & $\begin{array}{l}\text { Yokobori (2) } 2013 \\
\text { Yokobori (4) } 2013\end{array}$ & 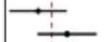 & $2.19(1.10,4.34)$ & 11.72 \\
\hline Shimada (2) 2012 & $i \cdot-$ & $4.47(1.54,18.95)$ & 4.86 & $\begin{array}{l}\text { Yokobori (4) } 2013 \\
\text { Shimada (2) } 2012\end{array}$ & 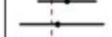 & $\begin{array}{l}4.37(2.15 .8 .94) \\
3.50(1.42 .10 .54)\end{array}$ & $\begin{array}{l}10.87 \\
5.50\end{array}$ \\
\hline linuma(1-2) 2011 & $\rightarrow$ & $2.62(1.14,4.20)$ & 18.02 & linuma(1-2) 2011 & $\div-$ & $\begin{array}{l}3.50(1.42,10.54) \\
3.26(1.74,6.25)\end{array}$ & $\begin{array}{l}5.50 \\
13.50\end{array}$ \\
\hline linuma(2-2) 2011 & 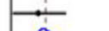 & $2.47(1.06 .6 .25)$ & 9.74 & linuma(2-2) 2011 & - & $2.97(1.44,6.40)$ & 9.92 \\
\hline Subtotal (1-squared $=22.1 \%, p=0.268$ ) & $\phi$ & $2.96(1.96,4.47)$ & 64.35 & Subtotal (l-squared $=29.0 \%, p=0.217$ ) & $\infty$ & $2.83(1.92,4.19)$ & 53.82 \\
\hline Overall (1-squared $=0.0 \%, p=0.651$ ) & $\dot{0}$ & $3.15(2.38 .4 .15)$ & 100.00 & Overall (1-squared $=0.0 \%, p=0.769$ ) & $\dot{\phi}$ & $3.03(2.40,3.83)$ & 100.00 \\
\hline NOTE: Weights are trom random oftects anaysis & i & & & NOTE. Weights are trom ransom etrects anaysis & & & \\
\hline .00563 & 1 & 178 & & .0248 & 1 & 40.3 & \\
\hline${ }_{{ }^{10}}^{\text {Study }} \mathbf{E}$ & & HR $(95 \% \mathrm{Cl})$ & $\begin{array}{l}\% \\
\text { Weight }\end{array}$ & ${ }_{\text {ID }}^{\text {Study }} \mathbf{F}$ & & $\operatorname{HR}(95 \% \mathrm{Cl})$ & $\begin{array}{l}\% \\
\text { Weight }\end{array}$ \\
\hline Stage II & $\vdots$ & & & Stage II & & & \\
\hline $\begin{array}{l}\text { Liu (1) } 2013 \\
\text { Yokobori (1) } 2013\end{array}$ & بـ & $0.77(0.13,4.62)$ & 2.33 & $\begin{array}{l}\text { Liu (1) } 2013 \\
\text { Yokobori (1) } 2013\end{array}$ & - & $0.50(0.13,2.01)$ & 2.71 \\
\hline $\begin{array}{l}\text { Yokobori (1) } 2013 \\
\text { Yokobori (3) } 2013\end{array}$ & $\bar{\vdots}$ & $4.07(1.50,11.57)$ & 7.12 & $\begin{array}{l}\text { Yokobori (1) } 2013 \\
\text { Yokobori (3) } 2013\end{array}$ & 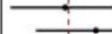 & $2.73(1.16,6.24)$ & 7.19 \\
\hline $\begin{array}{l}\text { Yokobori (3) } 2013 \\
\text { Shimada (1) } 2012\end{array}$ & $\square$ & $4.60(1.34,16.36)$ & 4.75 & $\begin{array}{l}\text { Yokobori (3) } 2013 \\
\text { Shimada (1) } 2012\end{array}$ & 二 & $4.46(1.73,11.62)$ & 5.61 \\
\hline $\begin{array}{l}\text { Shimada (1) } 2012 \\
\text { linuma(1-1) } 2011\end{array}$ & 广 & $9.82(1.99,177.57)$ & 1.47 & $\begin{array}{l}\text { Shimada (1) } 2012 \\
\text { linuma(1-1) } 2011\end{array}$ & 二 & $3.60(1.45,10.86)$ & 5.03 \\
\hline $\begin{array}{l}\text { linuma }(1-1) 2011 \\
\text { linuma }(2-1) 2011\end{array}$ & ! & $3.21(1.44,7.46)$ & 10.98 & linuma(1-1) 2011 & - & $3.35(1.66,6.74)$ & 10.37 \\
\hline $\begin{array}{l}\text { linuma(2-1) } 2011 \\
\text { Koch 2006 }\end{array}$ & $i$ & $3.75(1.25,12.47)$ & 5.62 & $\begin{array}{l}\text { linuma }(2-1) 2011 \\
\text { Koch 2006 }\end{array}$ & ב.i & $\begin{array}{l}3.10(1.33,7.24) \\
2.40(1.30 .530)\end{array}$ & 7.09 \\
\hline $\begin{array}{l}\text { Koch } 2006 \\
\text { Subtotal (1-squared }=0.0 \%, p=0.599 \text { ) }\end{array}$ & 8 & $\begin{array}{l}6.40(2.20,34.00) \\
3.72(2.36,5.85)\end{array}$ & $\begin{array}{l}3.96 \\
36.24\end{array}$ & $\begin{array}{l}\text { Koch } 2006 \\
\text { Subtotal (1-squared }=22.4 \%, p=0.258 \text { ) }\end{array}$ & $\Leftrightarrow$ & $\begin{array}{l}2.40(1.30,5.30) \\
2.77(1.90,4.02)\end{array}$ & $\begin{array}{l}10.31 \\
48.31\end{array}$ \\
\hline Stage III & & & & Stage III & & & \\
\hline Liu (2) 2013 & 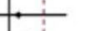 & $1.37(0.28,6.78)$ & 2.93 & Liu (2) 2013 & & $1.83(0.61,5.52)$ & 4.20 \\
\hline Yokobori (2) 2013 & $\rightarrow$ & $2.57(1.42 .4 .63)$ & 21.28 & Yokobori (2) 2013 & $\longrightarrow$ & $2.19(1.10,4.34)$ & 10.80 \\
\hline Yokobori (4) 2013 & 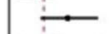 & $7.01(2.80,19.41)$ & 7.93 & Yokobori (4) 2013 & & $4.37(2.15,8.94)$ & 10.02 \\
\hline Shimada (2) 2012 & $\div$ & $4.47(1.54,18.95)$ & 4.71 & Shimada (2) 2012 & : & $3.50(1.42,10.54)$ & 5.08 \\
\hline linuma(1-2) 2011 & $\rightarrow$ & $2.62(1.14,4.20)$ & 17.48 & linuma(1-2) 2011 & $\longrightarrow$ & $3.26(1.74,6.25)$ & 12.45 \\
\hline $\begin{array}{l}\text { linuma(2-2) } 2011 \\
\text { Subtotal }(1-\text { squared }=0.0 \%, p=0.432)\end{array}$ & $\ddot{8}$ & $\begin{array}{l}2.47(1.06 .6 .25) \\
2.94(2.09,4.14)\end{array}$ & $\begin{array}{l}9.44 \\
63.76\end{array}$ & $\begin{array}{l}\text { linuma(2-2) } 2011 \\
\text { Subtotal }(1-\text { squared }=0.0 \%, p=0.729)\end{array}$ & $\infty$ & $\begin{array}{l}2.97(1.44,6.40) \\
3.00(2.19,4.11)\end{array}$ & $\begin{array}{l}9.15 \\
51.69\end{array}$ \\
\hline Overall (1-squared $=0.0 \%, p=0.608$ ) & $\phi$ & $3.20(2.44,4.21)$ & 100.00 & Overall (1-squared $=0.0 \%, p=0.560$ ) & $\phi$ & $2.90(2.32,3.64)$ & 100.00 \\
\hline NOTE: Weights are from rannoom efrects anayysis & 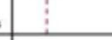 & & & NOTE: Weights are from ransom effocts analysis & & & \\
\hline .00563 & 1 & 178 & & .0861 & 1 & 11.6 & \\
\hline
\end{tabular}

prognostic information with approximately 93\% 5-year stage-specific survival rate for stage I, $84 \%$ for stage II, and $83 \%$ for stage III [40] and is influenced by clinicopathological parameters such as vascular invasion, poor differentiation, tumor size and serum tumor markers (i.e., carcinoembryonic antigen, CEA). Recently, many molecular biomarkers and high-risk gene signatures have been demonstrated to provide further information 


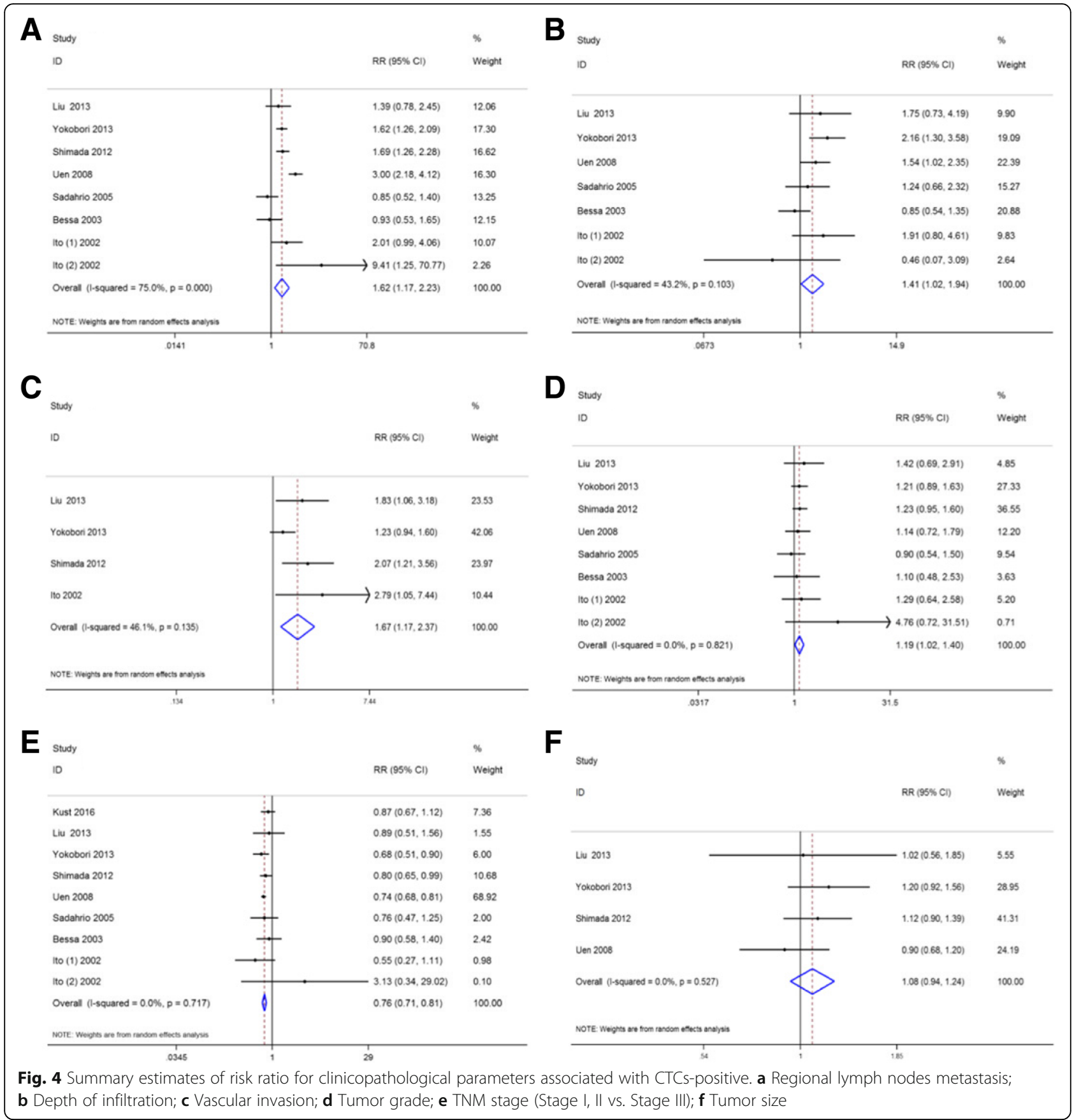

to support clinical decisions, however, none were conclusively accurate to evaluate the prognosis of all patients.

Since CTCs were first identified in PB of CRC patients, the clinical value of CTCs had become a debated topic throughout the medical community. From the clinical perspective, CTC analyses has an advantage in terms of a cost and ease of operation to serve as a monitoring tool pre and post treatments. Numerous studies had demonstrated that CTCs detection could provide important prognostic information for patients with CRC. A previous meta-analysis by Groot et al. had demonstrated the prognosis significance of detection of CTCs in patients with mCRC [41]. Peach et al. reviewed the prognostic value of postoperative detection of CTCs in non-mCRC patients and concluded that the presence of CTCs in PB was an independent predictor of recurrence [42]. However, the two meta-analyses were limited by the presence of methodological heterogeneity; the included studies used several different methods to detect CTCs and were not stratified 
Table 3 Results of subgroup analyses on OS

\begin{tabular}{|c|c|c|c|c|}
\hline Variables & $\mathrm{HR}[95 \% \mathrm{Cl}]$ & Number & $P^{2}$ & $P^{d}$ \\
\hline \multicolumn{5}{|l|}{ Year $>$ median $^{\mathrm{a}}$} \\
\hline Yes & $3.65[2.49-5.36]$ & 6 & $0.00 \%$ & 0.578 \\
\hline No & $2.44[1.19-4.99]$ & 7 & $72.90 \%$ & 0.001 \\
\hline \multicolumn{5}{|l|}{ Country } \\
\hline East Asia & $3.39[2.27-5.05]$ & 10 & $46.50 \%$ & 0.051 \\
\hline Non-East Asia & $2.10[0.52-8.54]$ & 3 & $74.50 \%$ & 0.02 \\
\hline \multicolumn{5}{|l|}{ Marker } \\
\hline Single & $2.72[1.48-5.00]$ & 9 & $66.80 \%$ & 0.002 \\
\hline Multiple & $3.77[2.62-5.43]$ & 4 & $0.00 \%$ & 0.799 \\
\hline \multicolumn{5}{|c|}{ Sampling time point } \\
\hline Pre-op & $3.65[2.49-5.36]$ & 6 & $0.00 \%$ & 0.578 \\
\hline Intra/post-op & $2.44[1.19-4.99]$ & 7 & $72.90 \%$ & 0.001 \\
\hline \multicolumn{5}{|c|}{ Patient no. $>$ median $^{b}$} \\
\hline Yes & $3.45[2.57-4.65]$ & 6 & $0.00 \%$ & 0.801 \\
\hline No & $2.59[1.08-6.22]$ & 7 & $74.20 \%$ & 0.001 \\
\hline \multicolumn{5}{|c|}{ Detection rate $>$ mean $^{c}$} \\
\hline Yes & $1.57[0.42-5.79]$ & 4 & $74.10 \%$ & 0.009 \\
\hline No & $3.71[2.84-4.85]$ & 9 & $0.00 \%$ & 0.796 \\
\hline \multicolumn{5}{|l|}{ Quality of study } \\
\hline Low & $4.06[1.64-10.05]$ & 2 & $0.00 \%$ & 0.384 \\
\hline High & $2.95[1.87-4.65]$ & 11 & $61.50 \%$ & 0.004 \\
\hline Overall & $3.07[2.05-4.62]$ & 13 & $55.70 \%$ & 0.008 \\
\hline
\end{tabular}

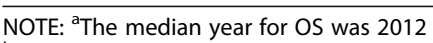

${ }^{\mathrm{b}}$ The median patient no. for OS was 103

'The mean detection rate for OS was $38.12 \%$

${ }^{\mathrm{d}}$ Two-tailed $P$ value of tests for heterogeneity

by detection method. With regard to the detection methods of CTCs, the prognostic utility of the CellSearch system in CRC patients had been demonstrated by a metaanalysis [43]. However, the clinical application of the RTPCR approach in the non-mCRC patients has still not been illustrated by a large-scale data analysis.

This study is the first comprehensive meta-analysis to validate the clinical significance of CTC detection by RT-

Table 4 Results of meta-regression on OS

\begin{tabular}{lllll}
\hline Variables & Coef. $^{a}$ & Std. Err. $^{\text {b }}$ & $P$ value & Adj R-squared $^{c}$ \\
\hline Year & 0.5072 & 0.4559 & 0.2900 & $0.67 \%$ \\
Country & 0.5352 & 0.5770 & 0.3740 & $1.08 \%$ \\
Marker & 0.4133 & 0.5021 & 0.4280 & $-11.42 \%$ \\
Time point & -0.5072 & 0.4559 & 0.2900 & $0.67 \%$ \\
Patient no. & 0.3751 & 0.4688 & 0.4410 & $-10.13 \%$ \\
Detection rate(mean) & -1.1526 & 0.4288 & 0.0210 & $93.80 \%$ \\
Quality of study & -0.3412 & 0.7123 & 0.6410 & $-12.93 \%$ \\
\hline
\end{tabular}

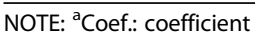

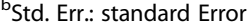

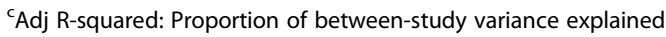

PCR method only in non-mCRC. The results demonstrated that CTC-positive patients had poorer OS and DFS than CTCs-negative patients at different sampling time (pre-OP and intra/post-OP), TNM stage (II/III) and adjuvant therapy status (without/post-OP chemotherapy), indicating that the clinical prognosis of patients with non-mCRC is significantly associated with the CTCs detected by RT-PCR in PB. Our pooled analyses also assessed the association between CTCs and clinicopathological parameters of non-mCRC patients and showed that CTC-positive was correlated with regional lymph nodes metastasis, deep depth of tumor infiltration, vascular invasion, poor differentiation of tumor and later TNM stage. Moreover, all these parameters have been shown to be an indicators of poor prognosis in CRC patients. Combined with the results of our collective evaluation, CTC-positive in $\mathrm{PB}$ has been demonstrated to be considered a prognostic and predictive marker for patients with non-mCRC. Numerous studies have demonstrated that there was not relationship between tumor size and the positivity of CTCs detection $[28,35]$; the results of our study were consistent with these previous studies.

Although we limited the studies included in our metaanalysis to those that used RT-PCR to reduce the heterogeneity caused by the difference in detection methods, no significant heterogeneity was found in the pooled analysis of DFS $\left(\mathrm{I}^{2}=34.0 \%, P=0.085\right)$. Nevertheless, there was still a certain extent of heterogeneity in our meta-analysis. Especially for OS, heterogeneity was mainly caused by data from the study by Shimada et al. [19]. Heterogeneity may also come from differences in the year, country and quality of publication, along with differences in sampling time, detection marker, or detection rate. Differences in the experimental designs in the cohort studies also generated non-negligible heterogeneity. To explore the potential sources of heterogeneity, subgroup analyses were performed based on year, country and quality of publication, sampling time, marker, number of patients, or detection rate, but the results were inconclusive (Table 3). Further, the results of the meta-regression clarified the heterogeneity and showed the detection rate was mainly responsible for the heterogeneity on OS. The detection rate of CTCs was greatly different based on different stage of early CRC. Stage I was too low, however, and the CTC-positive rate was significantly increased in stage III CRC patients, which had already been confirmed in studies using the CellSearch system $[14,15]$.

Theoretically, the association between prognosis and post-OP CTCs status was more convincing because post-OP CTCs status contains pre-OP CTCs and released CTCs during the operation [44]. However, the rapid apoptotic death of pre-OP CTCs may release mass tumor genes or antigens due to the change of the 

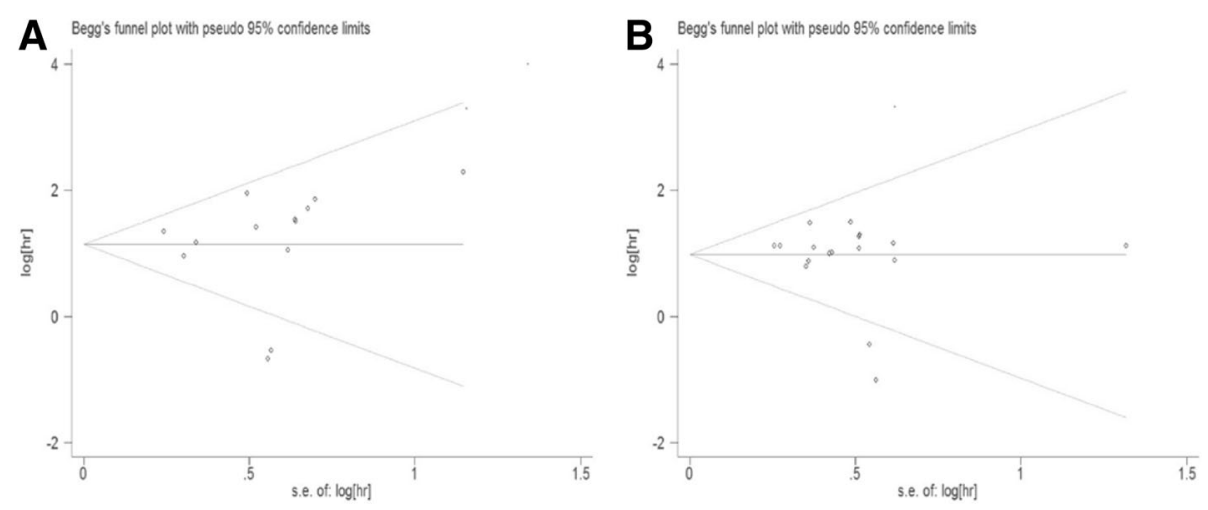

Fig. 5 Publication bias analysis. a Funnel plot of the studies on overall survival; b Funnel plot of the studies on disease-free survival

survival microenvironment in the process of operation, which might lead to a certain degree of detection bias. Therefore, the samples of post-OP samples could more accurately reflect the CTC status by including CTC release, apoptosis, and necrosis and could provide more information about the prognosis of patients. Ikeguchi $M$ et al. [45] found that in blood samples collected within $48 \mathrm{~h}$ after the operation, patients with CTC-positive had better prognosis than CTCsnegative patients. In our meta-analysis, the estimated result for OS remained stable and was not significantly affected by sampling time, which indicated CTCs detection not only at pre-OP but also post-OP could provide a prognostic factor. Thus, uncertainties still remain that sampling time could provide more accurate prognostic information, and further studies are needed to evaluate this relationship.

There were several limitations in our meta-analysis. First, our data for the meta-analysis came from previously published studies, and several included studies did not report HR. Therefore, we had to calculate them from the reported data with limited access to the raw data, which might affect the accuracy of the results. Second, there was considerable heterogeneity in our study. Although we eliminated the heterogeneity from detection methodology, RT-PCR cannot achieve CTCs enumeration and lacks biologic specificity. However, it does have the advantage of high sensitivity for CTCs detection [46]. We addressed the between-study heterogeneity by using a random effects model to obtain more conservative estimates. Third, language selection brings bias. We restricted the eligible studies to those written in English and excluded the relevant studies of other languages according to language criteria, which may cause language bias leading to an overestimation of effect sizes [47]. Despite these limitations, our meta-analysis is the first study to assess the prognostic significance of CTCs detected by RT-PCR in non-mCRC patients.
Our results provides an example for other studies that standardized testing method, optimized sampling time, complete analysis and report of results should be used to derive more accurate prognostic significance of CTCs in non-mCRC and CRC patients.

\section{Conclusions}

Based on available evidence, our meta-analysis suggested that the detection of CTCs in PB by RT-PCR is a prognostic factor for patients with non-mCRC, and CTC-positive was associated with poor prognosis and poor clinicopathological prognostic factors. However, the prognostic value of CTCs supports the use of CTCs as an indicator of metastatic disease prior to the current classification of mCRC meaning it is detectable by CT/MRI. Further highquality, well-designed, large-scale multicenter studies are required to evaluate the clinical significance and utility of CTCs detected by RT-PCR in non-mCRC patients.

\section{Abbreviations}

Cls: Confidence intervals; CRC: Colorectal cancer; CT: Chemotherapy; CTCs: Circulating tumor cells; DFS: Disease-free survival; HR: Hazard ratio; mCRC: Metastatic CRC; non-mCRC: Non-metastatic CRC; OP: Operative; OS: Overall survival; PB: Peripheral blood; RLNs: Regional lymph nodes; RR: Relative risk; RT-PCR: Reverse-transcriptase polymerase chain reaction; TNM: Tumor-node-metastasis

\section{Acknowledgements}

Not applicable

\section{Funding}

This work was supported by National Natural Science Foundation of China (No. 81572874). The funding body was not involved in the design of the study and collection, analysis, and interpretation of data and in writing the manuscript.

\section{Availability of data and materials}

The datasets supporting the conclusions of this article are included within the article.

\section{Authors' contributions}

$\mathrm{CY}$ and $\mathrm{KZ}$ contributed equally to this work. CY, KZ, and BX were responsible for conception and design of the study. CY and KZ did the studies selection, data extraction, statistical analyses and writing of the manuscript. LZ participated in studies selection and data extraction, and provided statistical expertise. CY, KZ and LZ contributed to the literature search, studies 
selection, and figs. CY, KZ and ZW provided clinical expertise and interpretation of data. The report was drafted, revised, and approved by all investigators. All authors read and approved the final manuscript.

\section{Ethics approval and consent to participate}

Not applicable

\section{Consent for publication}

Not applicable

\section{Competing interests}

The authors declare that they have no competing interests.

\section{Publisher's Note}

Springer Nature remains neutral with regard to jurisdictional claims in published maps and institutional affiliations.

\section{Author details}

'Department of Gastrointestinal Surgery \& Department of Gastric and Colorectal Surgical Oncology, Zhongnan Hospital of Wuhan University; Hubei Key Laboratory of Tumor Biological Behaviors \& Hubei Cancer Clinical Study Center, No.169 Donghu Road, Wuchang District, Wuhan 430071, China. 2Department of Oncology, Central Hospital of Wuhan, No.16 Gusaoshu Road, Jianghan District, Wuhan 430014, China.

\section{Received: 26 November 2016 Accepted: 25 October 2017}

\section{Published online: 07 November 2017}

\section{References}

1. Torre LA, Bray F, Siegel RL, Ferlay J, Lortet-Tieulent J, Jemal A. Global cancer statistics, 2012. CA Cancer J Clin. 2015:65(2):87-108.

2. Chen $W$, Zheng $R$, Baade PD, Zhang S, Zeng H, Bray F, Jemal A, Yu XQ, He J. Cancer statistics in China, 2015. CA Cancer J Clin. 2016;66(2):115-32.

3. Edwards BK, Ward E, Kohler BA, Eheman C, Zauber AG, Anderson RN, Jemal A, Schymura MJ, Lansdorp-Vogelaar I, Seeff LC, et al. Annual report to the nation on the status of cancer, 1975-2006, featuring colorectal cancer trends and impact of interventions (risk factors, screening, and treatment) to reduce future rates. Cancer. 2010;116(3):544-73.

4. Hayashi $M$, Inoue $Y$, Komeda $K$, Shimizu T, Asakuma M, Hirokawa F, Miyamoto Y, Okuda J, Takeshita A, Shibayama Y, et al. Clinicopathological analysis of recurrence patterns and prognostic factors for survival after hepatectomy for colorectal liver metastasis. BMC Surg. 2010;10:27.

5. Fidler IJ. The pathogenesis of cancer metastasis: the 'seed and soil' hypothesis revisited. Nat Rev Cancer. 2003;3(6):453-8.

6. Ashworth T. A case of cancer in which cells similar to those in the tumours were seen in the blood after death. Aust Med J. 1869:14(3):146-9.

7. Engell HC. Cancer cells in the circulating blood; a clinical study on the occurrence of cancer cells in the peripheral blood and in venous blood draining the tumour area at operation. Acta chirurgica Scandinavica Supplementum. 1955:201:1-70.

8. Pantel K, Alix-Panabieres C. Circulating tumour cells in cancer patients: challenges and perspectives. Trends Mol Med. 2010;16(9):398-406.

9. Tibbe AG, Miller MC, Terstappen LW. Statistical considerations for enumeration of circulating tumor cells. Cytometry Part A. 2007;71(3):154-62.

10. Paterlini-Brechot $P$, Benali NL. Circulating tumor cells (CTC) detection: clinica impact and future directions. Cancer Lett. 2007;253(2):180-204.

11. Seeberg LT, Waage A, Brunborg C, Hugenschmidt H, Renolen A, Stav I, Bjornbeth BA, Brudvik KW, Borgen EF, Naume B, et al. Circulating tumor cells in patients with colorectal liver metastasis predict impaired survival. Ann Surg. 2015;261(1):164-71

12. Aggarwal C, Meropol NJ, Punt CJ, lannotti N, Saidman BH, Sabbath KD, Gabrail NY, Picus J, Morse MA, Mitchell E, et al. Relationship among circulating tumor cells, CEA and overall survival in patients with metastatic colorectal cancer. Ann Oncol. 2013:24(2):420-8.

13. Sotelo MJ, Sastre J, Maestro ML, Veganzones S, Vieitez JM, Alonso V, Gravalos C, Escudero P, Vera R, Aranda E, et al. Role of circulating tumor cells as prognostic marker in resected stage III colorectal cancer. Ann Oncol. 2015:26(3):535-41.

14. Kuboki $Y$, Matsusaka S, Minowa S, Shibata H, Suenaga M, Shinozaki E, Mizunuma N, Ueno M, Yamaguchi T, Hatake K. Circulating tumor cell (CTC) count and epithelial growth factor receptor expression on CTCs as biomarkers for cetuximab efficacy in advanced colorectal cancer. Anticancer Res. 2013:33(9):3905-10.

15. Bork U, Rahbari NN, Scholch S, Reissfelder C, Kahlert C, Buchler MW, Weitz J, Koch M. Circulating tumour cells and outcome in non-metastatic colorectal cancer: a prospective study. Br J Cancer. 2015;112(8):1306-13.

16. Zhe $X$, Cher ML, Bonfil RD. Circulating tumor cells: finding the needle in the haystack. Am J Cancer Res. 2011;1(6):740-51.

17. Riethdorf S, Fritsche H, Muller V, Rau T, Schindlbeck C, Rack B, Janni W, Coith C, Beck K, Janicke F, et al. Detection of circulating tumor cells in peripheral blood of patients with metastatic breast cancer: a validation study of the CellSearch system. Clin Cancer Res. 2007;13(3):920-8.

18. Allard WJ, Matera J, Miller MC, Repollet M, Connelly MC, Rao C, Tibbe AG, Uhr JW, Terstappen LW. Tumor cells circulate in the peripheral blood of all major carcinomas but not in healthy subjects or patients with nonmalignant diseases. Clin Cancer Res. 2004;10(20):6897-904.

19. Shimada $R$, linuma $H$, Akahane $T$, Horiuchi $A$, Watanabe T. Prognostic significance of CTCS and CSCs of tumor drainage vein blood in Dukes' stage B and C colorectal cancer patients. Oncol Rep. 2012;27(4):947-53.

20. Kust D, Samija I, Kirac I, Radic J, Kovacevic D, Kusic Z. Cytokeratin 20 positive cells in blood of colorectal cancer patients as an unfavorable prognostic marker. Acta Clin Belg. 2016:71(4):235-43.

21. Tierney JF, Stewart LA, Ghersi D, Burdett S, Sydes MR. Practical methods for incorporating summary time-to-event data into meta-analysis. Trials. 2007:8:16.

22. Smith T, Smith B, Ryan MA. Survival analysis using Cox proportional hazards modeling for single and multiple event time data. In: Proceedings of the twenty-eighth annual SAS users group international conference, SAS Institute, inc, Cary, paper; 2003. p. 228-54.

23. Stang A. Critical evaluation of the Newcastle-Ottawa scale for the assessment of the quality of nonrandomized studies in meta-analyses. Eur J Epidemiol. 2010;25(9):603-5.

24. Schmidt FL, Oh IS, Hayes TL. Fixed- versus random-effects models in metaanalysis: model properties and an empirical comparison of differences in results. The British journal of mathematical and statistical psychology. 2009; 62(Pt 1):97-128

25. Higgins JP, Thompson SG. Quantifying heterogeneity in a meta-analysis. Stat Med. 2002;21(11):1539.

26. Egger M, Davey Smith G, Schneider M, Minder C. Bias in meta-analysis detected by a simple, graphical test. BMJ. 1997;315(7109):629-34.

27. Begg CB, Mazumdar M. Operating characteristics of a rank correlation test for publication bias. Biometrics. 1994;50(4):1088-101.

28. Liu Y, Qian J, Feng JG, Ju HX, Zhu YP, Feng HY, Li DC. Detection of circulating tumor cells in peripheral blood of colorectal cancer patients without distant organ metastases. Cell Oncol. 2013;36(1):43-53.

29. Yokobori T, linuma $H$, Shimamura $T$, Imoto $S$, Sugimachi $K$, Ishii $H$, Iwatsuki M, Ota D, Ohkuma M, Iwaya T, et al. Plastin3 is a novel marker for circulating tumor cells undergoing the epithelial-mesenchymal transition and is associated with colorectal cancer prognosis. Cancer Res. 2013;73(7):2059-69.

30. Iinuma H, Watanabe T, Mimori K, Adachi M, Hayashi N, Tamura J, Matsuda K, Fukushima R, Okinaga K, Sasako M, et al. Clinical significance of circulating tumor cells, including cancer stem-like cells, in peripheral blood for recurrence and prognosis in patients with Dukes' stage B and C colorectal cancer. J Clin Oncol. 2011:29(12):1547-55.

31. Uen YH, CY L, Tsai HL, FJ Y, Huang MY, Cheng TL, Lin SR, Wang JY. Persistent presence of postoperative circulating tumor cells is a poor prognostic factor for patients with stage I-III colorectal cancer after curative resection. Ann Surg Oncol. 2008;15(8):2120-8.

32. Barreto G, Neve R, Shrikhande SV, Shukla PJ. Role of circulating tumour cells in predicting recurrence after excision of primary colorectal carcinoma ( $\mathrm{Br}$ J Surg 2007; 94: 96-105). Br J Surg. 2007;94(5):644. author reply 644-645

33. Koch M, Kienle P, Kastrati D, Antolovic D, Schmidt J, Herfarth C, von Knebel Doeberitz M, Weitz J. Prognostic impact of hematogenous tumor cell dissemination in patients with stage II colorectal cancer. Int J Cancer. 2006; 118(12):3072-7.

34. Lloyd JM, Mclver CM, Stephenson SA, Hewett PJ, Rieger N, Hardingham JE. Identification of early-stage colorectal cancer patients at risk of relapse postresection by immunobead reverse transcription-PCR analysis of peritoneal lavage fluid for malignant cells. Clin Cancer Res. 2006;12(2):417-23.

35. Sadahiro S, Suzuki T, Ishikawa K, Saguchi T, Maeda Y, Yasuda S, Makuuchi H, Yurimoto S, Murayama C. Detection of carcinoembryonic antigen messenger RNA-expressing cells in portal and peripheral blood during 
surgery does not influence relapse in colorectal cancer. Ann Surg Oncol. 2005;12(12):988-94.

36. Bessa X, Pinol V, Castellvi-Bel S, Piazuelo E, Lacy AM, Elizalde JI, Pique JM, Castells A. Prognostic value of postoperative detection of blood circulating tumor cells in patients with colorectal cancer operated on for cure. Ann Surg. 2003;237(3):368-75

37. Ito S, Nakanishi H, Hirai T, Kato T, Kodera Y, Feng Z, Kasai Y, Ito K, Akiyama S, Nakao A, et al. Quantitative detection of CEA expressing free tumor cells in the peripheral blood of colorectal cancer patients during surgery with realtime RT-PCR on a LightCycler. Cancer Lett. 2002;183(2):195-203.

38. Treanor D, Quirke P. Pathology of colorectal cancer. Clin Oncol. 2007; 19(10):769-76.

39. Sargent D, Sobrero A, Grothey A, O'Connell MJ, Buyse M, Andre T, Zheng Y, Green E, Labianca R, O'Callaghan C, et al. Evidence for cure by adjuvant therapy in colon cancer: observations based on individual patient data from 20,898 patients on 18 randomized trials. J Clin Oncol. 2009;27(6):872-7.

40. Gunderson LL, Jessup JM, Sargent DJ, Greene FL, Stewart A. Revised tumor and node categorization for rectal cancer based on surveillance, epidemiology, and end results and rectal pooled analysis outcomes. J Clin Oncol. 2010;28(2):256-63.

41. Groot Koerkamp B, Rahbari NN, Buchler MW, Koch M, Weitz J. Circulating tumor cells and prognosis of patients with resectable colorectal liver metastases or widespread metastatic colorectal cancer: a meta-analysis. Ann Surg Oncol. 2013;20(7):2156-65.

42. Peach G, Kim C, Zacharakis E, Purkayastha S, Ziprin P. Prognostic significance of circulating tumour cells following surgical resection of colorectal cancers: a systematic review. Br J Cancer. 2010;102(9):1327-34.

43. Huang X, Gao P, Song Y, Sun J, Chen X, Zhao J, Xu H, Wang Z. Metaanalysis of the prognostic value of circulating tumor cells detected with the CellSearch system in colorectal cancer. BMC Cancer. 2015;15:202.

44. Weitz J, Kienle P, Lacroix J, Willeke F, Benner A, Lehnert T, Herfarth C, von Knebel Doeberitz M. Dissemination of tumor cells in patients undergoing surgery for colorectal cancer. Clin Cancer Res. 1998;4(2):343-8.

45. Ikeguchi M, Kaibara N. Detection of circulating cancer cells after a gastrectomy for gastric cancer. Surg Today. 2005;35(6):436-41.

46. Wicha MS, Hayes DF. Circulating tumor cells: not all detected cells are bad and not all bad cells are detected. J Clin Oncol. 2011:29(12):1508-11.

47. Pham B, Klassen TP, Lawson ML, Moher D. Language of publication restrictions in systematic reviews gave different results depending on whether the intervention was conventional or complementary. J Clin Epidemiol. 2005;58(8):769-76.

\section{Submit your next manuscript to BioMed Central and we will help you at every step:}

- We accept pre-submission inquiries

- Our selector tool helps you to find the most relevant journal

- We provide round the clock customer support

- Convenient online submission

- Thorough peer review

- Inclusion in PubMed and all major indexing services

- Maximum visibility for your research

Submit your manuscript at www.biomedcentral.com/submit

) Biomed Central 